\title{
Abnormal Kinetics of the Redox Reactions of Intrinsic Mitochondrial Pyridine Nucleotides in Hypothyroidism ${ }^{1}$
}

Thyroid hormone participates in oxidative phosphorylation, as shown by measurements of

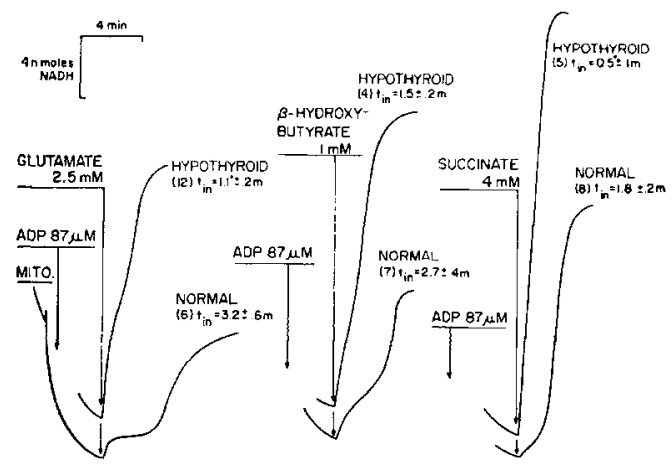

FIG. 1. Kinetics of the redox states of pyridine nucleotides in liver mitochondria from hypothyroid rats, compared to those from normal rats. Medium: $0.25 \mathrm{~m}$ sucrose; $21.1 \mu \mathrm{M}$ EDTA; $10.9 \mathrm{mM}$ $\mathrm{KCl} ; 10.9 \mathrm{~mm}$ Tris buffer; $4.23 \mathrm{~mm} \mathrm{P}_{\mathrm{l}}$; mitochondria in $0.25 \mathrm{~m}$ sucrose, $1.5 \mathrm{mg}$ protein; volume $2.0 \mathrm{ml}, \mathrm{pH} 7.4,25^{\circ} \mathrm{C}$. Protein was measured by a rapid biuret reaction (7). Fluorescence is measured (6) as a function of time, and the scale is calibrated by adding standard aliquots of NADH. The intramitochondrial pyridine nucleotides are brought to an oxidized state by adding $87 \mu \mathrm{M}$ ADP, then either glutamate, $\beta$-hydroxybutyrate, or succinate is added. Each curve was obtained on mitochondria from one or two rats, and chosen as average for the group; the duration of the inflection ( $t_{i n}$ in $\min$ ) observed in the reductive phase is measured by extrapolation between the steepest parts of the curve, and the number of experiments, the mean value, and the standard error are shown; the asterisk indicates $P<0.05$ for the comparison of hypothyroids and normals.

mitochondrial function $(1,2)$ and iodine content (3-5). We have observed an abnormality in an energy-dependent component in the redox kinetics

1 These studies were supported by grants from the NIH (AM 13564) and The John A. Hartford Foundation. Preliminary experiments were done in 1967 in collaboration with Dr. Peter F. Curran in the Biophysical Laboratory, Harvard Medical School. of intramitochondrial pyridine nucleotides in intact respiring mitochondria obtained from the livers of hypothyroid rats.

Mitochondria were prepared in $0.25 \mathrm{M}$ sucrose, using either normal fasted male rats, or littermates made hypothyroid as previously described (1). The relative degree of reduction of the in-
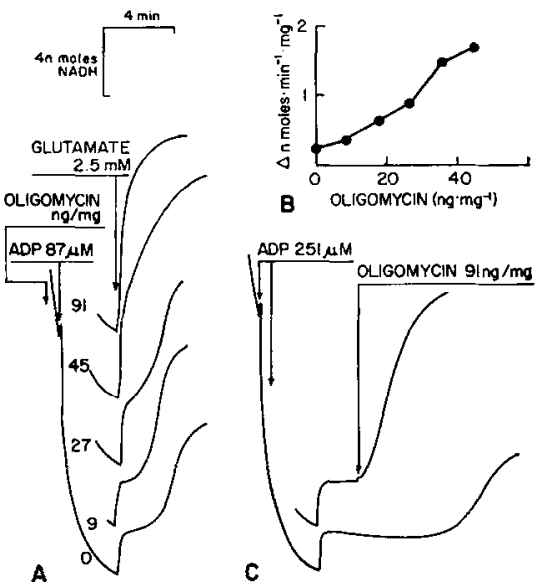

FIG. 2. Kinetics of the redox states of pyridine nucleotides in liver mitochondria from normal rats, and the action thereon of oligomycin. Experimental conditions are as in Fig. 1. (A) The intramitochondrial pyridine nucleotides, in the presence of increasing amounts of oligomycin, are brought to the oxidized state by adding ADP, and then to the reduced state by adding glutamate. (B) The slopes of the inflections that occur after glutamate is added in (A) are plotted as a function of oligomycin concentration. (C) The sequence of additions is $251 \mu \mathrm{M} \mathrm{ADP}$, glutamate, toligomyoin.

trinsic pyridine nucleotide in a suspension was measured fluorimetrically as a function of time after addition of $\mathrm{ADP}+\mathrm{P}_{\mathrm{i}}$ and then a substrate (6). In mitochondria from the hypothyroid rats, glutamate, $\beta$-hydroxybutyrate, or succinate produce a twice-normal difference between the highly oxidized steady state $\left(+\mathrm{ADP}+\mathrm{P}_{\mathrm{i}}\right.$ ) and the final highly reduced state (Fig. 1). This difference is consistent with the reported doubling of pyridine nucleotide content in mitochondria from hypothyroid rats (8). The normal delay in the substrate-induced reductive phase of the kinetics is 
markedly shortened in hypothyroidism, as measured by the duration of the inflection. Adding oligomycin to the hypothyroid mitochondria does not further accelerate the reductive phase (not shown in figure), but removes the inflection and increases the amplitude of the redox change in normal mitochondria (Fig. 2).

The delay in the reductive phase in normal mitochondria has been ascribed to a rate-controlling pyridine nucleotide transhydrogenase reaction (6), in the direction NADH + NADP $^{+} \rightarrow$ $\mathrm{NAD}^{+}+\mathrm{NADPH}$. This reaction is now recognized to be energy dependent (9). The energy-dependent component of the kinetic curve seems to be accelerated in hypothyroidism. Oligomycin, acting as a negative effector for phosphorylation processes, mimics the effects of thyroid hormone deficiency in this system. These observations suggest that the thyroid hormone may affect the availability of high-energy intermediates for specific energy-conserving or -wasting mitochondrial processes, rather than the amount of an intermediate that is generally available to several such processes (10).

\section{REFERENCES}

1. НосH, F. L. (1968). Arch. Biochem. Biophys. $124,238$.
2. Hoch, F. I. (1968). Arch. Biochem. Biophys. $124,248$.

3. Carr, E. A., JR., and RigGs, D. S. (1953). Biochem. J. 54, 217.

4. Drllon, R. S., And Hoch, F. L. (1967). Biochem. Med. 1, 219.

5. Hoch, F. L., and Motta, M. V. (1968). Proc. Nat. Acad. Sci. U.S.A. 59, 118.

6. Klingenberg, M., and Slenczka, W. (1959). Biochem. $Z$. 331, 486.

7. Gornall, A. G., Bardawill, C. J., AND David, M. W. (1949). J. Biol. Chem. 177, 751.

8. Kadenbach, B. (1966). Biochem. $Z$. 344, 49.

9. Danielson, L., and Ernster, L. (1963). Biochem. Z. 338, 188.

10. Носн, F. L. (1971). Energy Transformations in Mammals: Regulatory Mechanisms (Masoro, E. J., ed.), p. 88, Saunders, Philadelphia, Pennsylvania.

Frederic L. Hoch

The Departments of Internal Medicine

and Biological Chemistry

The University of Michigan Medical School

Ann Arbur, Michigan 48104

Received February 18, 1972; accepted, March 7, 197\% 